\title{
Moringa protects against nicotine-induced morphological and oxidative damage in the frontal cortex of Wistar rats
}

\author{
Ismail Temitayo Gbadamosi, Gabriel Olaiya Omotoso, Olayemi Joseph Olajide, \\ Shakirat Opeyemi Dada-Habeeb, Tolulope Timothy Arogundade, Ezra Lambe, \\ Kosisochukwu Kingsley Obasi \\ Department of Anatomy, Faculty of Basic Medical Sciences, College of Health Sciences, University of Ilorin, Ilorin, Kwara State, Nigeria
}

\begin{abstract}
The use of nicotine-containing substances has been implicated in oxidative-induced neuronal damage in several neurological dysfunctions. This study assessed the antioxidant potentials of Moringa tea on the frontal cortex of Wistar rats. Twenty female Wistar rats were divided into 4 groups of 5 animals each. Group A (control) received normal saline, Group B received $5.71 \mathrm{mg} / \mathrm{kg}$ of Moringa tea, Group C was treated with $13.76 \mathrm{mg} / \mathrm{kg}$ nicotine, while Group D received $5.71 \mathrm{mg} / \mathrm{kg}$ of Moringa tea and 13.76 $\mathrm{mg} / \mathrm{kg}$ nicotine, for 21 days. Homogenate of excised frontal cortex of rats obtained on day 22 was used to assess the level of malondialdehyde, catalase, superoxide dismutase and glutathione peroxidase. Histological sections were stained with heamatoxylin and eosin. Results showed increased activities of malondialdehyde and catalase in group C and a slight increase in group D compared with the control, while the activity of superoxide dismutase and glutathione peroxidase was reduced. The histological sections showed a normal architecture of the frontal cortex of rats treated with Moringa tea, but disrupted morphology in the group treated with Moringa tea and nicotineand further distortion in those that received nicotine only, when compared with the control group. These results suggest that Moringa tea may reduce the oxidative stress associated with nicotine consumption and limit the extent of structural damage in the frontal cortex of Wistar rats.
\end{abstract}

Keywords: frontal cortex; Moringa tea; nicotine; oxidative damage

Anatomy 2016;10(3):170-176 (2016 Turkish Society of Anatomy and Clinical Anatomy (TSACA)

\section{Introduction}

Nicotine is one of the principal components of tobacco; other constituents include many toxins and carcinogens such as tar, polycyclic aromatic hydrocarbon, heavy metals, carbon monoxide, arsenic and hydrogen cyanide that are linked to various diseases in the body. ${ }^{[1,2]}$ The commonest source of nicotine is through cigarette smoking the practice of burning tobacco and inhaling the smoke. ${ }^{[3]}$ Nicotine replacement therapy (NRT) is used to decrease withdrawal symptoms triggered by smoking cessation in individuals who want to quit smoking and thus avoid the harmful effects of smoking and chewing tobacco. ${ }^{[4]}$ Underlying the supposed connection between nicotine and cognitive enhancement is the role of nicotinic acetylcholine receptors (nAChRs) in attention, learning, mem- ory, and cortical plasticity. ${ }^{[5]}$ nAChRs normally bind endogenous neurotransmitter acetylcholine, but are also particularly responsive to nicotine. They are abundant in brain regions associated with learning and memory, including the frontal cortex,${ }^{[6]}$ and in primate and rodent models, depletion of acetylcholine in the prefrontal cortex results in impaired attentional performance. ${ }^{[5]}$ Nicotine replacement products are most beneficial for heavy smokers who smoke more than 15 cigarettes per day. There are not adequate studies to show that NRT helps those who smoke fewer than 10 cigarettes per day. ${ }^{[7]}$

Moringa oleifera commonly known as drumstick or horseradish tree,$^{[8]}$ is indigenous to the Northwestern part of India, but also widely distributed in the tropics, West Africa and Central America as well as the 
Caribbean. ${ }^{[9]}$ Various parts of the tree have been used traditionally for the treatment of diabetes, rheumatism, hepatotoxicity, renal diseases and a variety of other diseases. $^{[9-11]}$ Given its therapeutic advantages, Moringa leaves have been processed into tea bags for easy consumption. The aim of the present study was to determine the effect of Moringa tea on oxidative stress markers and histoarchitecture of the frontal cortex following nicotine administration.

\section{Materials and Methods}

A total of 20 adult female Wistar rats with an average weight of $185 \pm 3.32 \mathrm{~g}$ were used for the study. Following the approval of the Ethics Committee of the University of Ilorin, the animals were housed in a wire gauzed cage in the animal house of the Faculty of Basic Medical Sciences at the University of Ilorin. The animals were allowed to acclimatize for two weeks prior to the commencement of the study.

The animals were divided into four groups (A-D) of five animals each. Group A was orally treated with $1 \mathrm{ml}$ of distilled water, Group B was treated with $5.71 \mathrm{mg} / \mathrm{kg}$ body weight of Moringa tea, Group C was treated with 13.76 $\mathrm{mg} / \mathrm{kg}$ nicotine in $0.1 \mathrm{ml}$ of vehicle once daily as the maximum tolerated dose in an earlier study, ${ }^{[12]}$ while Group D was treated with $5.71 \mathrm{mg} / \mathrm{kg}$ body weight of oral Moringa tea and $13.76 \mathrm{mg} / \mathrm{kg}$ nicotine i.p. once daily. All groups were treated for 21 consecutive days. Morinaga oleifera leaves were obtained and identified at the Department of Plant Biology of the University of Ilorin, Kwara State, Nigeria. Following weeks of sundrying, an aqueous extraction of the dry Moringa oleifera leaves was made and concentrated. Rats were weighed at 7-day intervals, beginning from day one of administration.

$24 \mathrm{~h}$ after the final administration of Morinaga oleifera, animals for histology were euthanized using $20 \mathrm{mg} / \mathrm{kg}$ of ketaminei.p. andperfused transcardially with normal saline, followed by $4 \%$ paraformaldehyde (PFA). The brains were excised and post-fixed for 24 hin $4 \%$ PFA and processed manually for haematoxylin and eosin stain. Rats processed for enzymatic studies were sacrificed by cervical dislocation to eliminate the meddling of ketamine-induced change in biochemical status. The brains were excised following proper decapitation and dissection, and place in $30 \%$ sucrose solution. The frontal cortices of the right and left lobes of each animal were obtained and then homogenized manually with $30 \%$ sucrose solution. Each homogenate was centrifuged at $3000 \mathrm{rpm}$ for $10 \mathrm{~min}$ and the supernatant was extracted for further enzymatic analysis. Enzymatic studies were carried out using the enzyme linked immunosorbent assay.
The results obtained from enzymatic analysis were subjected to statistical analysis using the GraphPad Prism software, Version 6 (GraphPad Software Inc., San Diego, CA, USA). Malondialdehyde (MDA), glutathione peroxidase $(\mathrm{GSH})$, catalase (CAT) and superoxide dismutase (SOD) results were plotted in one way ANOVA with Tukey's multiple comparisons test. Data obtained were presented as mean \pm standard error of mean, with determination of level of significance at $p$ value less than 0.05 . The outcomes were represented in bar charts with error bars to show the mean and standard error of mean, respectively.

\section{Results \\ Body weight changes}

The body weights of the animals were obtained on days 1 , 7, 14 and 21. The animals in the nicotine group were observed to reduce the amount of daily food intake. Statistical analysis of the body weight of the animals revealed that the body weight of animals in the control group increased linearly over the period of treatment. There was an initial decrease in the body weight of animals treated with Moringa tea, but the weight gradually increased. Weight changes in the nicotine-treated group continuously decreased. Animals co-treated with nicotine and Moringa tea had reduced weight initially, followed by steady body weight over the period of administration (Figure 1 and 2).



Figure 1. Linear graph showing changes in body weight over the period of administration. Line A: control (Group A); Line B: Moringa tea (Group B); Line C: nicotine (group C), and Line D: nicotine and Moringa tea (Group D). Group A showed a continuous linear increase in weight, while group B showed an initial subtle decrease and then a continuous increase in body weight. Group $C$ had a continuous linear decrease in weight, but Group D showed mild decrease until day 7, and thereafter weight was fairly stable. 


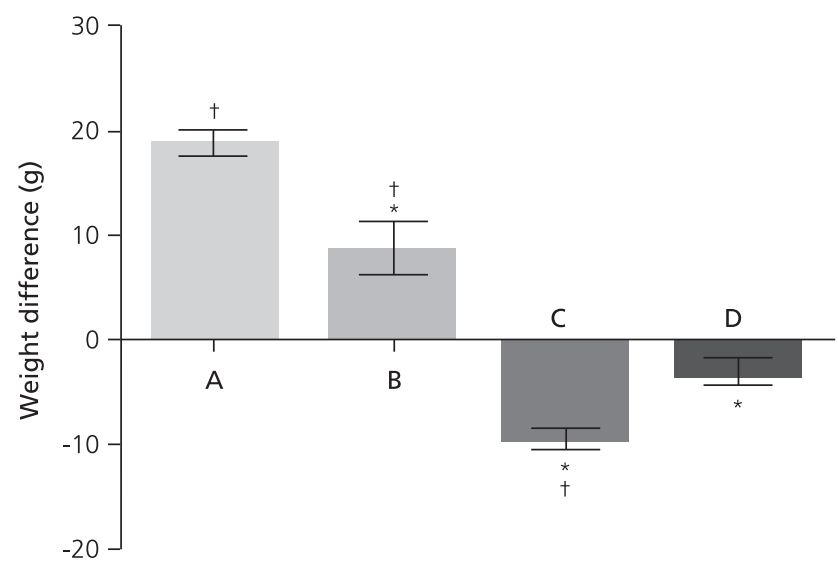

Figure 2. Changes in body weight. A: control; B: Moringa tea; C: nicotine and $\mathrm{D}$ : nicotine and Moringa tea. ${ }^{*} \mathrm{p}<0.05$ compared with the control; ${ }^{\dagger} p<0.05$ compared with Group D. Group B showed a significantly lesser weight gain, while Groups $C$ and D showed significant weight loss when compared to $\mathrm{A}$. C shows a significantly higher weight loss when compared to $\mathrm{D}$.

\section{Biochemical analysis}

The biochemical data obtained in this study for each group which include MDA, GSH, CAT and SODare shown in Figures 3-6. The results showed increased MDA and CAT activities in group $\mathrm{C}$ and a slight increase in group D compared to group A, while reverse was the case for SOD and GSH.

The histological sections of the frontal cortices of saline-treated and Moringa-treated animals showed a proper and normal cortical neuronal cells layout from the outermost molecular layer to the innermost multiform layer. The nicotine-treated frontal cortices showed

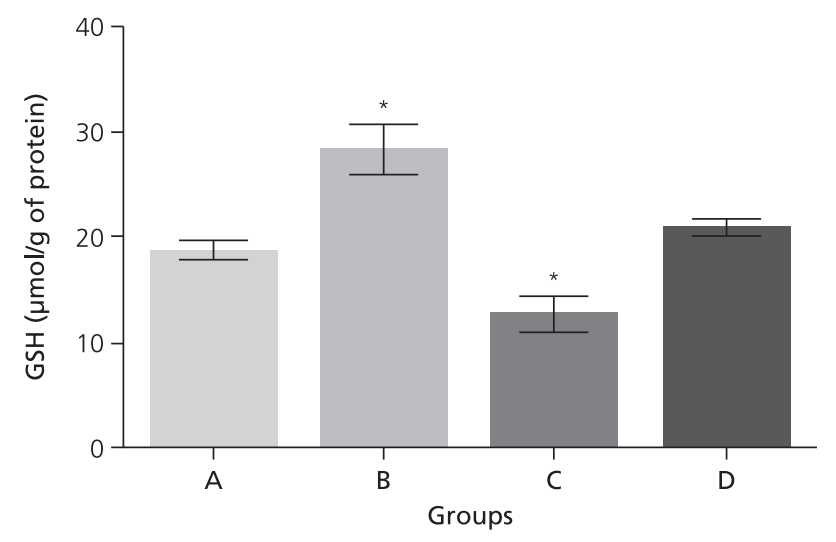

Figure 4. Graph showing the changes in the levels of GSH. A: control; $\mathrm{B}$ : Moringa tea; C: nicotine and D: nicotine and Moringa tea. ${ }^{*} \mathrm{p}<0.05$ in comparison with the control group. Group B showed significant increase and Group $C$ significant decrease, while there was no significant change in the GSH level in Group D.

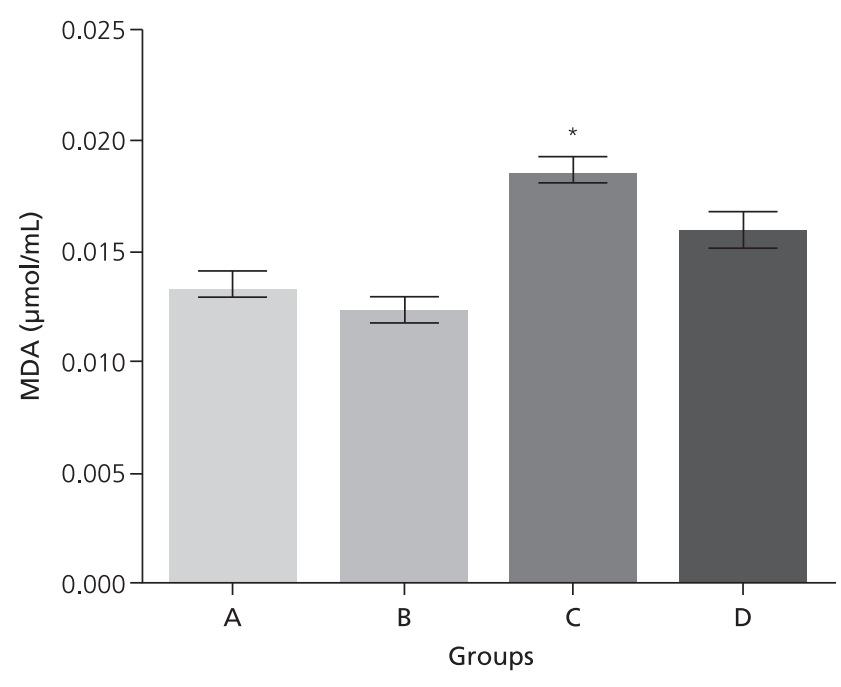

Figure 3. Graph showing the changes in the levels of MDA. Figure 4. Graph showing the changes in the levels of GSH. A: control; B: Moringa tea; C: nicotine and D: nicotine and Moringa tea. ${ }^{*} p<0.05$ in comparison with the control group. Group B showed significant increase and Group C significant decrease, while there was no significant change in the MDAlevel in Group D.

a distorted cellular layout. The extent of distortion in the animals treated with combined Moringa and nicotine was limited when compared to the nicotine-treated animals. The increase in MDA levels was also indicated in (Figure 7) that showed intact cell membrane in A and B, but poor demarcation in $\mathrm{C}$ due to the degraded lipid bilayer of the neuronal and non-neuronal cell membranes. The conspicuous delineation in the frontal cortex of animals Group D can be attributed to the neuroprotective effects of Moringa to on the neuronal cells against the neurodegenerative tendencies of nicotine.

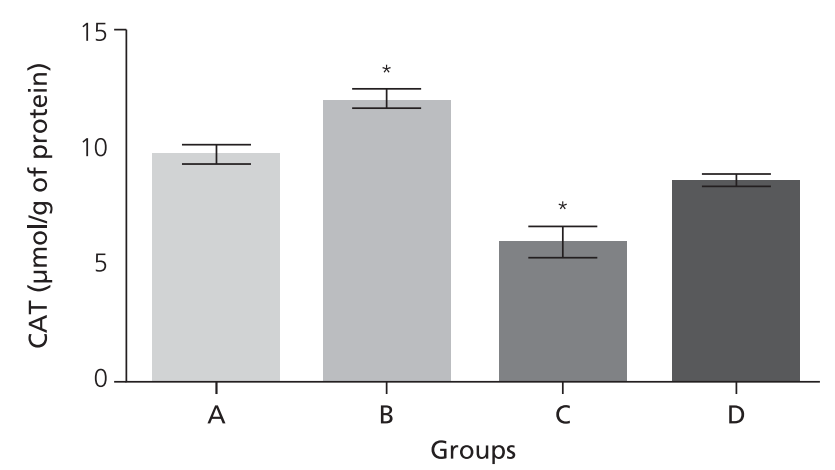

Figure 5. Graph showing the changes in the levels of CAT. A: control; B: Moringa tea; C: nicotine and D: nicotine and Moringa tea. ${ }^{*} p<0.05$ in comparison with the control group. Group B showed significant increase and Group C significant decrease, while there was no significant change in the CAT level in Group D. 
In the internal granular layer of dorsolateral frontal cortex of Wistar rats treated with normal saline and Moringa tea, nuclei of the neuronal and non-neuronal were eosinophilic and deeply-stained with small pyramidal cells characterized by apical and basal dendrites sparsely distributed among the dominating stellate (granule) cells (Figure $\mathbf{8 a}, \mathbf{b})$. In rats treated with nicotine, neurons were apoptotic with poorly stained nuclei (Figure 8c). Histology of Moringa tea treatment in nicotine rats showed an improvement with healthy granule cells and few in apoptotic stages (Figure 8d).

\section{Discussion}

Nicotine is known to decrease insulin sensitivity ${ }^{[13]}$ and cause significant reduction in body weight of animals. There is a significant reduction in adipose tissue, particularly white fat masses, as fat deposits might be used as an

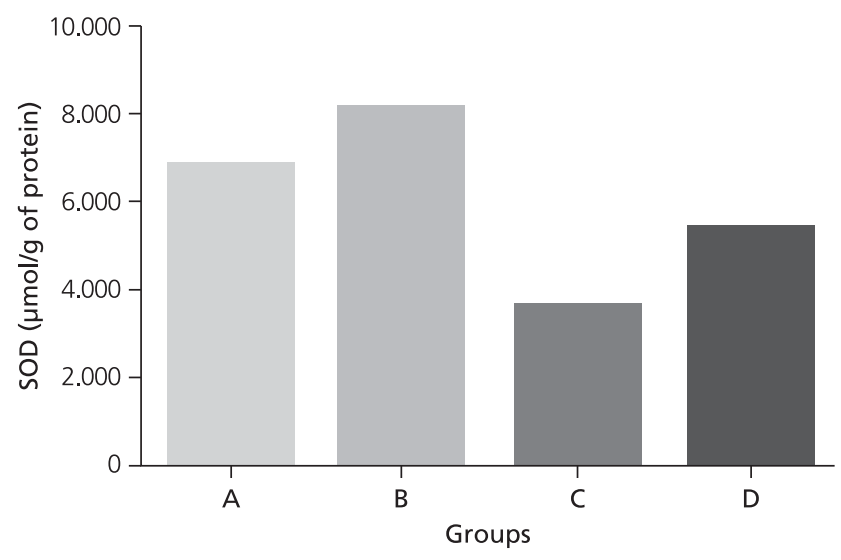

Figure 6. Graph showing the changes in the levels of SOD. A: control; B: Moringa tea; C: nicotine, and D: nicotine and Moringa. ${ }^{*} p<0.05$ in comparison with the control group. Group B showed significant increase and Group C significant decrease, while there was no significant change in the SOD level in Group D.


Figure 7. Photomicrographs showing the dorsolateral frontal cortex of Wistar rats treated with normal saline (a), Moringa tea (b), nicotine (c), and Moringa tea with nicotine (d). a (control) and b show normal cortical cell distribution, from the molecular layer (layer I) to the multiform layer (layer 6) with adequate and typical cellular density in the internal molecular and internal pyramidal layer (L3 and L4). c shows distorted cell distribution and poor cellular delineation more than found in $\mathbf{d}$. Density and size of neurons were reduced in $\mathbf{c}$ and $\mathbf{d}$ in comparison to $\mathbf{a}$ and $\mathbf{b}$. Perineural spaces and fragmented neuropils (yellow dotted circles) were noticeable in $\mathbf{d}$. Neuronal cells were laconically expressed in $\mathbf{d}$. Heamatoxylin and eosin stain $\times 100$. [Color figure can be viewed in the online issue, which is available at www.anatomy.org.tr] 
energy supply under these conditions of negative energy balance. ${ }^{[14]}$ The pattern of change in body weight of animals in the control group showed a continuous linear increase over the period of administration. Animals in the Moringa-treated group showed an initial slight decrease in body weight after which there was a linear progression in the body weight. The nicotine-treated group experienced a continuous decrease in body weight over the period of administration. This pattern of continuous weight loss experienced by animals in the nicotine-treated group was reversed by the actions of Moringa in the group treated synergistically with nicotine and Moringa.

Food intake in all the treated animals was reduced compared with the control animals, although Perkins et al. ${ }^{[15]}$ reported no difference in total caloric intake or micronutrient or taste selection in smokers and non- smoker. Also, the work of Winders and Grunberg ${ }^{[16]}$ reported that nicotine administration produced no comparable cumulative effect on growth rate even though he reported changes in food consumed and body weight. The findings in the present study corroborate some earlier works that substantial weight loss was detected in animals exposed to cigarette smoke, which was a factor of reduction in food intake. ${ }^{[17-20]}$

Nicotine affects a variety of cellular processes ranging from secretion of hormones to modulation of enzymatic activities ${ }^{[8]} \mathrm{MDA}$ is a marker of lipid peroxidation, and in the present study, its levels increased significantly in the nicotine group as well as in the group treated concurrently with Moringa tea and nicotine, with animals given nicotine only having a higher level. Nicotine increases MDA levels in the vascular wall of rat off-
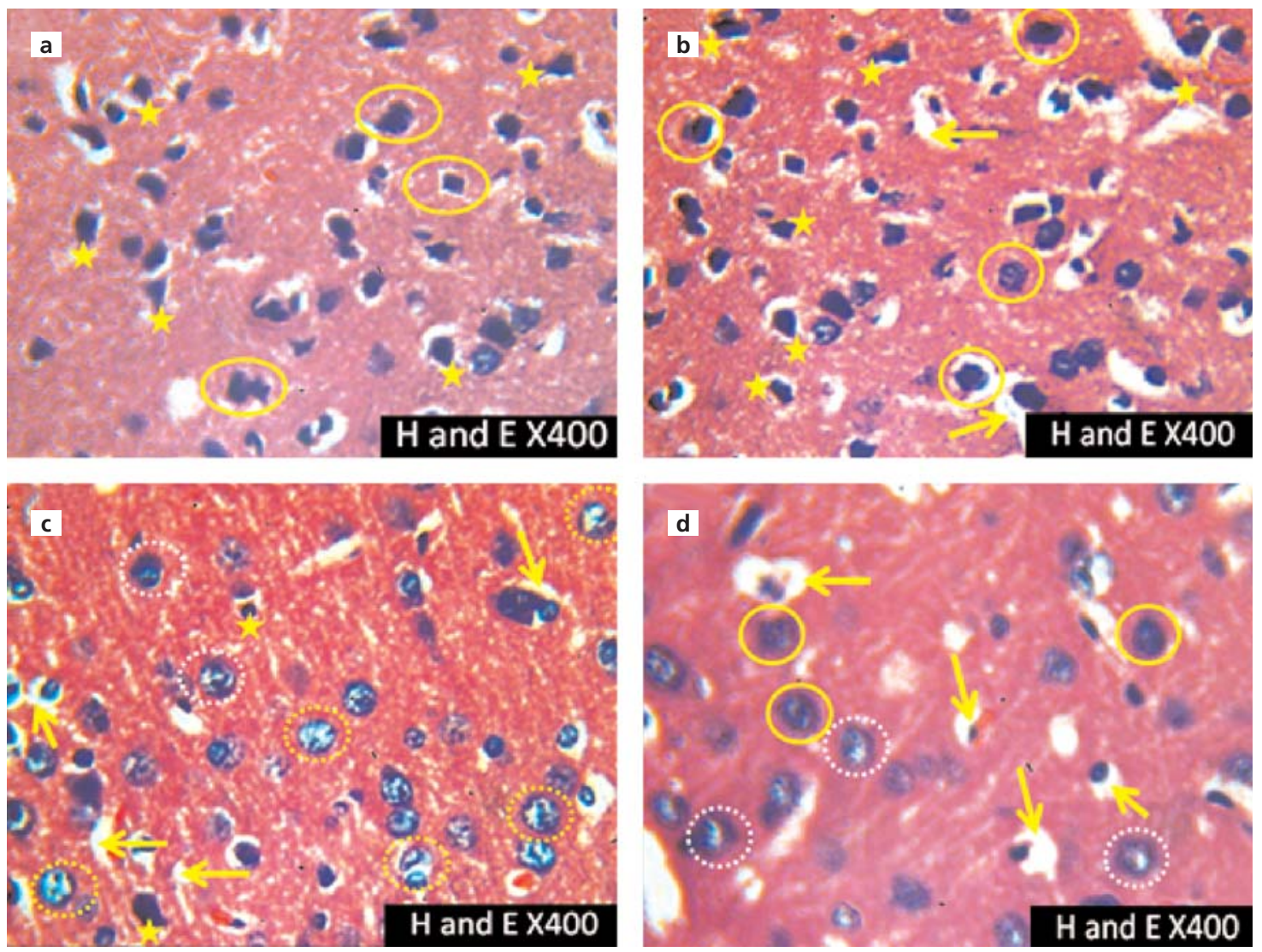

Figure 8. Photomicrographs of the internal granular layer of dorsolateral frontal cortex of Wistar rats treated with normal saline (a), Moringa tea (b), nicotine (c), and Moringa tea with nicotine (d). a and b show small pyramidal cells characterized by apical and basal dendrites (yellow stars) sparsely distributed among the dominating stellate (granule) cells (yellow circles) characterized by their several dendritic processes. Nuclei of the neuronal and non-neuronal cells found in $\mathbf{a}$ and $\mathbf{b}$ are adequately eosinophilic and deeplystained. Many of the granule cells in c are in their early apoptotic (white dotted circles) or late apoptotic (yellow dotted circles) stages characterized by their poorly stained nuclei. $\mathbf{d}$ is marked by healthy granule cell (yellow circles) and few granule cells in their early apoptotic stages (white dotted circles). $\mathbf{c}$ and $\mathbf{d}$ express perineural spaces (yellow arrows) which are predominant in $\mathbf{c}$ (Heamatoxylin and eosin stain $\times 100$ ). [Color figure can be viewed in the online issue, which is available at www.anatomy.org.tr] 
spring $^{[21]}$ and maternal foetal brain tissues. ${ }^{[8]}$ The expression of NADPH oxidase increases following nicotine treatment, which is linked to an increase in $\mathrm{O}^{2-}$ production and oxidative damage of the vasculature as indicated by the increase in MDA levels. ${ }^{[21]}$

A significant increase and decrease in the levels of catalase was observed in Moringa-treated and the nicotine-treated groups. However, the level of decrease was not significant in rats administered with both Moringa tea and nicotine. This observation was due to the counterbalancing actions of Moringa tea against that of the nicotine that was administered to animals in this group.

The level of GSH increased significantly in the group treated with Moringa tea unlike the nicotine-treated group which had a significant decrease. There was an increase in the level of GSH in the group that received the combined treatment of Moringa tea and nicotine, but the level of increase was not significant. The partial increase in the levels of GSH in the group given Moringa tea and nicotine compared to the decrease in nicotine-treated group could be attributed to the effect Moringa tea that was concomitantly administered to the former group.

A significant increase in the levels of SOD was observed in Moringa-treated animals compared with the nicotine-treated rats. The reduced level of SOD in animals that received the combination of Moringa tea and nicotine, though not significant, could be ascribed to the compensating actions of Moringa against nicotine. This observation is in agreement with previous report by Xioa et al. (2011) that nicotine treatment significantly decreased the level of the antioxidant enzyme activities such as SOD. ${ }^{[21]}$

In the current study, assessment of the level of MDA showed that treatment with Moringa tea counterbalanced the neurotoxic effect of nicotine. This can be related from Figures 3-6 that showed a decrease in the level of MDA and increase in the levels of SOD, GSH and CAT in the group that was treated in tandem with Moringa tea and nicotine. The extremely defensive effects of Moringa oleifera against nicotine-induced neurotoxicity has been sustained by the findings of Gupta et al. who demonstrated its therapeutic efficacies. ${ }^{[13]}$ The present study is also in line with studies by Luqman et al. ${ }^{[2]}$ who reported that Moringa decreased lipid oxidation and increased antioxidant activity in mice. Faiza ${ }^{[8]}$ also suggested that the protective effects of Moringa oleifera extract on glutathione and MDA concentration may be attributed to the presence of phytoconstituents that scavenge free radicals, activate the antioxidant enzymes and inhibit oxidases. Moringa oleifera may decrease oxidative stress by other mechanisms such as the decreased oxidative stress generation capacity through the mitochondrial or inflammatory mechanisms. ${ }^{[23]}$

The extent of distortion observed in the animals treated with combined Moringa and nicotine was limited when compared to the nicotine-treated animals. This may be attributed to the presence of Moringa tea, as well as the increase in lipid peroxidation marked by the raised levels of MDA in the nicotine-treated group. Nicotine increases the levels of free radicals in, precisely $\mathrm{O} 2$ - which scavenges electrons from the lipids of the cell membrane of the neuronal cells. ${ }^{[24-26]}$

\section{Conclusion}

The use of Moringa tea may reduce the morphological alterations and oxidative damage associated with nicotine-induced neurotoxicity in the frontal cortex. This could offer clinical benefits in neurological dysfunctions associated with consumption of nicotine-containing substances.

\section{References}

1. Secretan B, Straif k, Baan R, Grosse Y, Ghissassi F, Buovard,V, Benbrahim-Tallaa L, Guha N, Freeman C, Galichet L, Cogliano V. A review of human carcinogens - Part E: tobacco, areca nut, alcohol, coal smoke and salted fish. Lancet Oncol 2009;10:1033-4.

2. Zainul Z. Dark nights behind the white clouds - risks of tobaccosmoking on human health besides the oral health and malignancy. EXCLI J 2011;10:69-85.

3. Gately I. Tobacco: a cultural history of how an exotic plant seduced civilization. New York (NY): Grove; 2001. p. 3-7.

4. Stead LF, Perera R, Bullen C, Mant D, Hartmann-Boyce J, Cahill $\mathrm{K}$, Lancaster $\mathrm{T}$. Nicotine replacement therapy for smoking cessation. Cochrane Database Syst Rev 2012;11:CD000146.

5. Wallace TL, Bertrand D. Importance of the nicotinic acetylcholine receptor system in the prefrontal cortex. Biochem Pharmacol 2013;85:1713-20.

6. Poorthuis RB, Goriounova NA, Couey JJ, Mansvelder HD. Nicotinic actions on neuronal networks for cognition: general principles and long-term consequences. Biochem Pharmacol 2009;78: 668-76.

7. Rigotti NA. Clinical practice. Treatment of tobacco use and dependence. N Engl J Med 2002;346:506-12.

8. Faiza A. Effect of Moringa oleifera extract on nicotine induced neurotoxicity in female rat and their embryos. Global Veterinaria 2013; 11:131-8.

9. Jaiswal D, Kumar PR, Kumar A, Mehta S, Watal G. Effect of Moringa oleifera Lam. leaves aqueous extract therapy on hyperglycemic rats. J Ethnopharmacol 2009;123:392-6.

10. Babu R, Chaudhuri M. Home water treatment by direct filtration with natural coagulant. J Water Health 2005;3:27-30.

11. Verma AR, Vijayakumar M, Mathela CS, Rao CV. In vitro and in vivo antioxidant properties of different fractions of Moringa oleifera leaves. Food ChemToxicol 2009;47:2196-201. 
12. Gabriel OO, Mercy OA, Abayomi AA, Josephine OI, Olalekan AO, Bernard UE. Neurohistochemical studies of adolescent rats' prefrontal cortex exposed to prenatal nicotine. Ibnosina Journal of Medicine and Biomedical Sciences 2014:6:1.

13. Gupta R, Kannan GM, Sharma M, S Flora SJ. Therapeutic effects of Moringa oleifera on arsenic-induced toxicity in rats. Environ Toxicol Pharmacol 2005;20:456-64.

14. Chen H, Vlahos R, Bozinovski S, Jones J, Anderson GP, Morris MJ. Effect of short-term cigarette smoke exposure on body weight, appetite and brain Neuropeptide Y in mice. Neuropsychopharmacology 2005; 30:713-9.

15. Perkins KA, Sexton JE, DiMarco A, Fonte C. Acute effects of tobacco smoking on hunger and eating in male and female smokers. Appetite 1994;22:149-58.

16. Winders SE, Grunberg NE. Effects of nicotine on body weight, food consumption and body composition in male rats. Life Sci 1990;46: 1523-30.

17. Albanes D, Jones DY, Micozzi MS, Mattson ME. Association between smoking and body weight in the US population: analysis of NHANES II. Am J Public Health 1987;77:439-44.

18. Bellinger L, Cepeda-Benito A, Wellman PJ. Meal patterns in male rats during and after intermittent nicotine administration. Pharmacol Biochem Behav 2003;74:495-504.
19. Bishop C, Parker GC, Coscina, DV. Systemic nicotine alters wholebody fat utilization in female rats. Physiol Behav 2004;80:563-7.

20. Omotoso GO, Babalola FA. Histological changes in the cerebelli of adult wistar rats exposed to cigarette smoke. Niger J Physiol Sci 2014;29:43-6.

21. Xiao D, Huang X, Yang S, Zhang L. Antenatal nicotine induces heightened oxidative stress and vascular dysfunction in rat offspring. Br J Pharmacol 2011;164:1400-9.

22. Luqman S, Srivastava S, Kumar R, Maurya AK, Chanda D. Experimental assessment of Moringa oleifera leaf and fruit for its antistress, antioxidant and scavenging potential using in vitro and in vivo assays. Evid Based Complement Alternat Med 2012;2012:519084.

23. Kirisattayakul W, Wattanathorn J, Tong-Un T, Muchimapura S, Wannanon P, Jittiwat J. Cerebroprotective effect of Moringa oleifera against focal ischemic stroke induced by middle cerebral artery occlusion. Oxid Med Cell Longev 2013;2013:951415.

24. Marnett LJ. Lipid peroxidation - DNA damage by malondialdehyde. Mutat Res 1999;424:83-95.

25. Zou XL, Chem JM. Serum SOD activity and lipid peroxidation. A handbook of heamatology from Wuhan University. 6th ed. Wuhan: Wuhan University Press; 2005. p. 43-71.

26. Michel F, Bonnefont-Rousselot D, Mas E, Drai J, Thérond P. Biomarkers of lipid peroxidation: analytical aspects. Ann Biol Clin (Paris) 2008; 66:605-20.

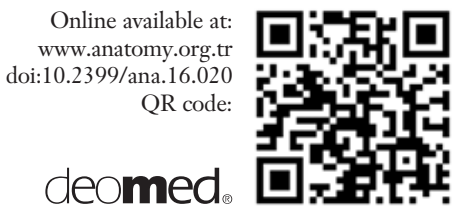

Correspondence to: Ismail Temitayo Gbadamosi

Department of Anatomy, Faculty of Basic Medical Sciences, College of Health Sciences, University of Ilorin, Ilorin, Kwara State, Nigeria

Phone: +234806 9031960

e-mail: ismail.tayo@yahoo.com

Conflict of interest statement: No conflicts declared.

This is an open access article distributed under the terms of the Creative Commons Attribution-NonCommercial-NoDerivs 3.0 Unported (CC BY-NC-ND3.0) Licence (http://creativecommons.org/licenses/by-nc-nd/3.0/) which permits unrestricted noncommercial use, distribution, and reproduction in any medium, provided the original work is properly cited. Please cite this article as: Gbadamosi IT, Omotoso GO, Olajide OJ, Dada-Habeeb SO, Arogundade TT, Lambe E, Obasi KK. Moringa protects against nicotine-induced morphological and oxidative damage in the frontal cortex of Wistar rats. Anatomy 2016;10(3):170-176. 